\title{
La influencia del pensamiento fenomenológico en el desarrollo de la disciplina etnográfica
}

\author{
Cristopher Valdés San Martín
}

Resumen

Esta investigación explora la relación epistemológica entre la antropología y la etnografía con la filosofía husserliana, desde una serie de autores que articulan las ideas de la fenomenología con los presupuestos de la etnografía, en especial, en lo concerniente al desarrollo de la noción de intersubjetividad. Una primera generación de autores incorpora de forma indirecta y parcial las bases epistemológicas y ontológicas de la propuesta filosófica de Husserl. Así, autores como Clifford Geertz, Maurice Natanson, Thomas Luckmann, Harold Garfinkel, Michael Agar y Thomas Csordas, construyeron parte de su pensamiento con la continuación de la fenomenología husserliana realizada por Merleau-Ponty y Alfred Schütz, utilizando los conceptos operatorios de ambos filósofos. Aun así, la profundidad y rigurosidad en el tratamiento de estos conceptos fenomenológicos por esta primera generación llevará a un manejo de la noción de intersubjetividad de forma más restringida y específica que los planteamientos realizados por Husserl. En los años 70 y 80, las influyentes y discutibles críticas realizadas por Bourdieu y Derrida a la filosofía husserliana tuvieron un importante eco en la antropología y la etnografía, propiciando el abandono del tratamiento directo de las ideas de Husserl. A pesar de esto, en las últimas dos décadas, autores como Michael Agar, Michael Jackson, Vincent Crapanzano, Alessandro Duranti, Jason Throop conforman una tercera generación que retoma las ideas husserlianas, logrando establecer una serie de dimensiones y aplicabilidades del concepto de intersubjetividad introducido y desarrollado por Husserl, que podrían servir de fundamento posible para un estudio de la condición humana y la cultura.

Palabras clave

Epistemología de la Ciencias Sociales; Intersubjetividad; Fenomenología; Etnografía

Códigos JEL: B0, Y9, Z0, Z1
THE INFLUENCE OF PHENOMENOLOGICAL THINKING IN THE DEVELOPMENT OF THE ETHNOGRAPHIC DISCIPLINE

Abstract

This research explores the epistemological relationship between anthropology and ethnography with Husserlian philosophy, from a series of authors who articulate the ideas of phenomenology with the presuppositions of ethnography, especially as regards the development of the notion of intersubjectivity. The first generation of authors indirectly and partially incorporate the epistemological and ontological bases of Husserl's philosophical proposal. Thus, authors such as Clifford Geertz, Maurice Natanson, Thomas Luckmann, Harold Garfinkel, Michael Agar, and Thomas Csordas, built part of their thinking with the continuation of the Husserlian phenomenology performed by Merleau-Ponty and Alfred Schütz, using the operative concepts of both philosophers. Even so, the depth and rigor in the treatment of these phenomenological concepts by this first generation will lead to a handling of the notion of intersubjectivity in a more restricted and specific way than the approaches made by Husserl. In the 70 s and $80 \mathrm{~s}$, the influential and debatable criticisms made by Bourdieu and Derrida to Husserlian philosophy, had an important echo in anthropology and ethnography, leading to the abandonment of the direct treatment of Husserl's ideas. Despite this, in the last two decades, authors such as Michael Agar, Michael Jackson, Vincent Crapanzano, Alessandro Duranti, Jason Throop, make up a third generation that takes up Husserlian ideas, managing to establish a series of dimensions and applications of the intersubjectivity concept introduced and developed by Husserl, which could serve as a possible basis for a study of the human condition and culture.

Keywords

Epistemology of Social Sciences; Intersubjectivity; Phenomenology; Ethnography

JEL codes: B0, Y9, Z0, Z1

Fecha de recepción del original: 24 de octubre de 2019; versión definitiva: 8 de septiembre de 2020 .

Cristopher Valdés San Martín. E-mail: valdes.sanmartin@gmail.com.

ORCID ID: 0000-0003-0856-038X. 


\section{La influencia del pensamiento fenomenológico en el desarrollo de la disciplina etnográfica ${ }^{1}$}

Cristopher Valdés San Martín

1. Introducción: fenomenología, intersubjetividad y etnografía

La finalidad de esta investigación no es establecer asociaciones de forma absoluta y cerrada del influjo de una corriente filosófica como la fenomenología en las ciencias sociales y, en especial, en la etnografía. Sería inapropiado reducir las fuerzas constructivas que han actuado en el desarrollo de la etnografía solo a un análisis unilineal. Más bien, vemos la necesidad de no desconocer las variadas vías de influencia ${ }^{2}$ epistemológica, ontológica y ética que han tenido impacto en las fuerzas constructivas de la etnografía hasta hoy, y focalizaremos nuestros esfuerzos en una de ellas: la fenomenología trascendental de Edmund Husserl y su noción de intersubjetividad, mediante el sentido e intención dado por varios autores que han realizado un puente entre su filosofía y las ciencias sociales. Cabe destacar que nuestra elección responde a la necesidad de volver a repensar los beneficios potenciales que puede ofrecer la fenomenología a la investigación antropológica (Throop y Murphy, 2002), donde la concepción de intersubjetividad presentada podría llegar a establecer una base común para todas las ramas de la antropología como la ciencia de la sociabilidad humana (Duranti, 2010). Veremos cómo las interpretaciones del pensamiento fenomenológico, desde las ciencias sociales y la etnografía, no han sido suficientemente exhaustivas y vale el esfuerzo de volver a revisar su pensamiento filosófico que po-

\footnotetext{
${ }^{1}$ El autor agradece a Bárbara Navarrete y Renato Valdés por el numen otorgado para realizar este artículo.

2 Desde el siglo XVIII, estas influencias han sido variadas en la etnografía, desde sus inicios como disciplina rigurosa con los trabajos de campo realizados por Gerhard Friedrich Müller, entre 1730 y 1740 en Asia septentrional (Vermeulen, 2015); o con un segundo hito situado para algunos autores en la $2^{\circ}$ expedición de Cambridge al Estrecho de Torres en Oceanía, realizado por William Halse Rivers Rivers (Guber, 2001: 25).
}

dría contribuir al conocimiento de un "otro" desde la mirada etnográfica.

Ahora bien, reconocer estas rutas de influencia desde la fenomenología hacia las ciencias del espíritu -o en sentido inverso-, presenta algunas dificultades que intentaremos sortear de la mejor manera. La primera es la heterogeneidad de enfoques epistemológicos que han conformado el desarrollo de las teorías antropológicas existentes en las ciencias sociales y, en específico, en la antropología, donde no hay contribución que pueda ser aislada sin tomar en consideración su contexto o corriente de pensamiento, estableciéndose una red de influencias, movimientos de ideas y relaciones de carácter unidireccionales o bidireccionales. La segunda problemática, enunciada por Maurice Merleau-Ponty, es lograr descubrir una influencia difusa no siempre querida por Husserl ni reconocida por los que la utilizan (Merleau-Ponty, 2011). Aunque varios reconocen esta deuda con la fenomenología trascendental de Husserl, revisaremos autores destacados de las ciencias sociales y la filosofía contemporánea que no lo hacen.

El camino que seguiremos para eludir estas dificultades será concentrarnos en uno de los principales ejes conceptuales de la antropología y la fenomenología trascendental de Edmund Husserl: Nos referimos al concepto de "intersubjetividad", su relevancia y orígenes, para después revisar qué autores de la filosofía realizan un puente entre la fenomenología y las ciencias sociales, particularmente a lo pertinente con la antropología y la etnografía.

\section{La noción de intersubjetividad husserliana}

Detrás de la reflexión antropológica y etnográfica sobre la concepción de la intersubjetividad en plano de la cultura, se 
entreteje una discusión intelectual de la concepción del mundo y la relación entre sujeto-investigador que han tenido diferentes modulaciones en su comprensión. Desde los padres de la antropología en la primera mitad del siglo XX (Boas, Malinowsky, Evans Pritchard), pasando por el trabajo de campo funcionalista, como también los aportes de Peter Winch a la comunidad antropológica de una teoría de la interpretación, han necesitado comprender las reglas intersubjetivas del lenguaje (Ulin, 1990). Posteriormente, en las décadas de los sesenta y setenta, la antropología crítica apunta contra sus antecesoras (el estructuralismo y el funcionalismo) acometiendo con una evaluación epistemológica del conocimiento, coincidiendo en la naturaleza intersubjetiva de los datos de campo, realizando una crítica a las nociones de verdad y certeza propuestas en las tradiciones del dualismo cartesiano (Wright, 2003: 350).

La centralidad de la intersubjetividad sigue estando presente en la mayor parte de la reflexión del conocimiento etnográfico y en sus formas de hacer, incluso de manera tácita. Eso no significa que exista una sola comprensión de la intersubjetividad, puesto que se establecen matices y densidades disimiles en los enfoques de pensamiento revisados.

¿Qué influencia epistemológica tiene la concepción de intersubjetividad utilizada en la antropología y la disciplina etnográfica?. La respuesta se debe situar históricamente en la génesis del concepto de intersubjetividad que no es parte del desarrollo de la teoría antropológica, sino que proviene de la filosofía contemporánea. Aunque el problema del "otro" -y nos referimos a "la necesidad intelectual de dar razón suficiente para explicar nuestra convivencia con otras personas"- ha estado presente en el pensamiento del ser humano moderno desde el cogito de Descartes (Laín, 1988). El concepto de intersubjetividad es introducido y luego elaborado por Edmund Husserl durante un período de tres décadas (Duranti, 2010: 1), convirtiéndose en el tema nuclear de su fenomenología (San Martín, 2015: 132) (Toledo, 2009: 73) que se origina con la elaboración de los constructos de "intersubjetividad trascendental" (Husserl, 1988: 15, 47, 49, 51) (Husserl, 1996: 76, 134, 141, 169, 197, 219, 227, 230), del "mundo intersubjetivo" (Husserl, 2013: 186, 457) y de la "objetividad intersubjetiva" (Husserl, 1997: 211, 245, 246) ${ }^{3}$. En el transcurso de su obra, Husserl frecuenta la revisión y reflexión de la intersubjetividad como un tema transversal de investigación que abarca todo el ámbito de experiencia humana y la sociabilidad de las personas (Duranti, 2010: 1-3). Estamos en presencia de una reflexión sistemática de la noción de intersubjetividad, que no solo abarca el problema del conocimiento, sino también el estatuto ontológico de la persona y su relación ético-histórica (San Martín, 1994: 157-188).

Husserl (1984) realiza la siguiente pregunta a las corrientes filosóficas antecesoras a su fenomenología: “¿No es entonces necesario que yo me pregunte cómo por sobre mi autoconciencia individual puedo poseer una conciencia general trascendental-intersubjetiva?". Busca comprender, a nivel de la percepción y del ser, la constitución recíproca entre una subje-

\footnotetext{
${ }^{3}$ Destacan los manuscritos de investigación de Husserl, obra no publicada en español, para una fenomenología de la intersubjetividad presentes en Husserliana XIII, XIV, XV, editados por Iso Kern (Velozo, 2006).
}

tividad individual para los seres humanos singularizados y una intersubjetivización de la comunidad humana.

“Esto se produce de tal modo que, en la conciencia de cada uno, y en la conciencia de la comunidad comprensiva que surge en la conexión, se hace valer constantemente y se conserva continuamente el mismo mundo en cuanto ya experimentado o como horizonte abierto de posibles experiencias de todos" (Husserl, 1984: 166).

El plano en el que trabaja la intersubjetividad es el de la conciencia, referida a la unidad de multiplicidad de experiencias propias y ajenas, lo que nos permite una vivencia compartida con el horizonte de los "otros", con los que se puede establecer una conexión actual o potencial. Uno y los "otros" nos encontramos referidos a las mismas cosas, donde cada uno capta distintos aspectos o perspectivas de los objetos, pero a partir del mismo sistema total de multiplicidad de cada uno. Todo en cuanto uno experimenta originaria y efectivamente, se convierte en mera "representación de" la cosa, que nadie ha visto ciertamente, ya que está en constante movimiento para la conciencia y las cambiantes experiencias propias y ajenas (Husserl, 1984).

En los diferentes horizontes de los seres humanos individuales o colectivos, actuales o potenciales, los que son abiertos e infinitos según los posibles encuentros en una vida en comunidad, se va estableciendo el contenido de la interculturalidad propia de la humanidad. El concepto de intersubjetividad en la fenomenología y la filosofía antropológica se convierte en interculturalidad ${ }^{4}$ y, si damos un paso más allá tomando en consideración sus pautas, podemos llegar a establecer un horizonte etnológico -cultural y mitológico- propio y particular de un pueblo (San Martín, 2015). El acceso a la intersubjetividad es la entrada a una multiplicidad de mundos posibles, o a uno de los posibles horizontes etnológicos que compartimos los grupos humanos, siendo central su comprensión epistemológica, ontológica y ética para la disciplina etnográfica, que tiene como finalidad el conocer a un "otro" desde un plano subjetivo, y encontrarnos en un "nosotros" que se da desde el fenómeno de la "Comunitarización" que constantemente hacemos.

En resumen, y siguiendo Alessandro Duranti, las dimensiones de la intersubjetividad incluyen a la relación humana y al mundo natural en el que vivimos, que normalmente damos por sentado (actitud natural), el cual es el objeto de nuestros pensamientos; el papel que juegan las herramientas y otros ar-

\footnotetext{
${ }^{4}$ Siguiendo a Javier San Martín, Husserl no plantea el concepto de interculturalidad, aunque si podemos sacar unas pautas en el terreno en que se da (San Martín, 2015: 141).

5 En la traducción de Hugo Steinberg de "La Crisis de las Ciencias Europeas y la Fenomenología Trascendental" en el numeral 47, se traduce el concepto "Vergemeinschaftung" como "Intersubjetivación". No obstante, en la traducción de la misma obra realizada por Julia Iribarne se traduce el mismo concepto como "Comunitarización". El Dr. Raúl Velozo Farías tradujo el concepto "Vergemeinschaftung" como "comunización" al traducir el texto de Husserl "La pregunta por el origen de la geometría como problema histórico-intencional" (Husserl, 1972). El fenómeno de la comunitarización es aquello donde los distintos egos se unen para cooperar en la constitución de un mundo único, así como, culturalmente, constituyen productos culturales como el único lenguaje compartido (Moran, 2012: 252).
} 
tefactos en la evocación de otros; el sentido de pertenencia a una comunidad o de una relación particular, aun cuando otros no son co-presentes; la participación en determinados tipos de encuentros sociales; el acceso y la utilización de los lenguajes humanos y otros recursos semióticos, al volver a conectar la lengua y los idiomas a una gama mucho más amplia de facultades y fenómenos que definen nuestra forma de estar en el mundo, apreciándose un amplio espectro de lo que entendemos como la "condición humana" (Duranti, 2010:11-14). En la actualidad visualizamos en las ciencias de la cultura un manejo del concepto de intersubjetividad de forma más restringida y específica que los planteamientos realizados por Husserl, respondiendo solo al sentido de "comprensión compartida o mutua" ${ }^{\prime 6}$ (shared or mutual understanding) de un otro (Duranti, 2010: 4).

La adopción de la intersubjetividad por la mayoría de otros estudiosos no incluyó necesariamente una discusión, una elaboración o una crítica de las ideas de Husserl. El resultado fue que, dentro de una o dos generaciones, el concepto de intersubjetividad llegó a ser separado del nombre de Husserl y se asentó una comprensión restringida, como es la noción de comprensión mutua o compartida. Desde una perspectiva psicológica, esta definición de la intersubjetividad como "entendimiento mutuo" apunta a "cómo las personas llegan a saber lo que otros tienen en mente y cómo se ajustan en consecuencia" (Bruner, 1996: 161). Y desde una mirada filosófica se concibe como "una estructura particular del discurso potencial" (Habermas, 1970: 373).

Ambas definiciones conciben la intersubjetividad como una potencialidad del conocimiento humano y no como lo había planteado Husserl, un a priori del conocimiento humano (Duranti, 2010: 10-14), estableciéndose una mirada epistemológica y ontológica más restringida de la "otredad" que la elaborada por Husserl o sus continuadores.

2.1. La influencia de Husserl en el pensamiento de Alfred Schütz y Merleau-Ponty.

Son muchos los autores que han tenido una influencia directa de Husserl en su obra y podríamos elaborar una lista de ilustres pensadores contemporáneos a él como: Martin Heidegger, Jean Paul Sartre, Emmanuel Levinas, Max Scheler, Eugene Minkowski, Ortega y Gasset, Edith Stein, Kurt Koffka; por dar algunos nombres que han impactado y siguen influenciando las diferentes disciplinas de las ciencias sociales. Pero, siguiendo el desarrollo de la idea de intersubjetividad, nos encontramos con dos autores que fueron inspirados por

\footnotetext{
${ }^{6}$ Podemos atribuir la popularidad de esta interpretación a una traducción al inglés de los términos en alemán "Wechselverständnis", "Einverständniss" y "Wechselverständingung" utilizados por Husserl en el primer volumen de Ideas (1913) (Duranti 2010: 5) y transferidos a otros autores de las ciencias sociales e incluso de la filosofía.
}

la fenomenología trascendental y han tenido un gran aporte al devenir de la antropología y la etnografía.

El primero es el austríaco Alfred Schütz, quien se basó en las concepciones posteriores de Husserl sobre la intersubjetividad y el "mundo de la vida" (Overgard y Zahavi, 2009) (Toledo, 2009: 73) para el desarrollo de un programa de investigación de los fenómenos sociales en ambos conceptos. El segundo es el autor francés Maurice Merleau-Ponty, quien reformula la concepción de intersubjetividad trascendental como intercorporeidad (López, 2004: 57), teniendo una influencia intelectual tangible en el desarrollo histórico de la antropología (Morris, 2015: 351). Ambos autores comparten varios elementos en común en el desarrollo de su pensamiento y obra que son importantes de revisar por su influencia epistemológica y ontológica para el desarrollo etnográfico, que no necesariamente significó un desarrollo fenomenológico.

Un primer punto en común es la reflexión en torno a la importancia de la relación recíproca entre filosofía y las ciencias humanas. Schütz indagó la contribución que la fenomenología puede hacer a los problemas metodológicos concretos en las ciencias sociales (Schütz, 1993: 127), teniendo en la base a una filosofía rigurosa que trianguló con la vertiente praxeológica de Ludwig von Mises, y la sociología comprensiva de Max Weber, haciendo avances hacia una sociología de la experiencia (Toledo, 2009: 74). Por su parte, Merleau-Ponty propone el desarrollo de una psicología y antropología que deben evitar una concepción empirista y racionalista al conocer a un "otro", debido a que ambas encarnan el error del "pensamiento objetivo" que es consistente en la idea de acceder a la realidad sin vernos afectados por nuestras interpretaciones y preocupaciones (Priest, 1998: 6). Ambos autores contribuyen a extender más allá de la filosofía el pensamiento fenomenológico, con ideas generales de relevancia para la antropología, sociología y psicología.

Una segunda similitud es la distinción entre los modos de existencia prereflexivos descritos en la fenomenología trascendental. Lo que Schütz llamó experiencia "pre-fenomenal" y Merleau-Ponty denominó "preobjetiva", las cuales son modos de existencia más reflexivos que surgen cuando tomamos actitudes teóricas hacia nuestras acciones, y las de otros, y pretenden desestabilizar aquellas suposiciones no examinadas que organizan nuestros compromisos prereflexivos con la "realidad" (Desjarlais y Throop, 2001: 88).

Un tercer punto de encuentro es compartir una mirada que rechaza las filosofías positivistas y reduccionistas del ser humano. Merleau-Ponty en su obra La estructura del comportamiento, publicada en 1942, critica las filosofías neopositivistas contemporáneas de la mente, argumentando que las negaciones ficticias de la realidad de la conciencia presuponen de hecho la conciencia (Priest, 1998: 2). También niega la tesis determinista de que existen condiciones causalmente suficientes para la obtención de estados mentales, ya que es la conciencia la que constituye algunas relaciones como causales. Por su parte, Schütz rechaza de manera enfática los programas reduccionistas, como el conductismo y positivismo, que intentan reducir la acción humana a un comportamiento observable de los mecanismos de estímulo y respuesta, debido a que el científico social debe construir modelos basados en 
agentes cotidianos que incluyen cosas como la conciencia, los motivos y la comprensión para hacer explícito el significado que tienen las estructuras y relaciones en los propios agentes observados (Overgard y Zahavi, 2009: 9).

2.2. La fenomenología de la intercorporalidad de Merleau-Ponty como referente epistemológico de la disciplina etnográfica.

Habría una unidad y coherencia de la reflexión merleaupontyana en todas sus fases que se apoya en el reconocimiento de un número de rasgos claves que definen el estilo de su entera filosofía (García, 2012: 12). El primer rasgo clave sería la incorporación de elementos de varias corrientes, manteniéndose fiel a la noción de que el sentido del objeto lingüístico debe comprenderse como la interrelación estructural de un número de elementos que no tienen existencia autónoma. Establecerá una interrelación entre intersubjetividad y lenguaje al establecer que al hablar y entender no son procesos que representen que se produce, sino que consisten, en lo que denomina María Carmen Lopéz, "inter- corporeidad” y una comunicación potencialmente universales (López, 1996: 212).

Otro rasgo transversal es una acción activa del cuerpo con el mundo y con los otros cuerpos, es decir, solo como un "inter-cuerpo". Por último, su adscripción al método fenomenológico, haciendo valer este punto de vista por sobre otras explicaciones de la percepción y la conducta. La filosofía husserliana le permitió a Merleau-Ponty encontrar un camino que no se opusiera al empirismo o el logicismo, sino que hiciera un camino entre ambos; un camino que revelará la tesis del mundo reafirmando la racionalidad en el nivel de las experiencias. Y es así como Merleau-Ponty (2011) se refiere a Husserl y el método:

"Necesita descubrir un modo de conocimiento que no sea el conocimiento deductivo y que tampoco sea el conocimiento simplemente empírico. Hace falta un conocimiento no-conceptual que no se separe del hecho y que, sin embargo, sea filosófico, o al menos que no vuelva imposible la existencia del sujeto filosofante" (Merleau-Ponty, 2011: 32)"

El método fenomenológico posibilitó a Merleau-Ponty eliminar toda especie de idealismo, poniendo fuera de juego y negando nuestra complicidad con el mundo por un instante, para después concebir al sujeto como trascendencia hacia el mundo (Merleau-Ponty, 1993: 13). Mediante un asombro ante el mundo, refiriéndose a la reducción fenomenológica como método, la reflexión realiza el siguiente juego:

"No se retira del mundo hacia la unidad de la conciencia como fundamento del mundo, toma su distancia para ver surgir sus trascendencias, distiende los hilos intencionales que nos vinculan al mundo para ponerlos de manifiesto" (Merleau-Ponty, 2011: 32).
Al revisar su obra, vemos que la adscripción al andamio conceptual fenomenológico no responde solo a la utilización de un método como salida del esencialismo, también hay una similitud de fondo que comparte con Husserl y reconoce como un problema transversal de la filosofía moderna y contemporánea la concepción del cogito cartesiano. Para Husserl, es el pensamiento de Descartes la fuente de separación del objetivismo físico y el subjetivismo trascendental (Biemel, 1968: 56). Merleau-Ponty pone en tela de juicio esta interpretación "eternitaria del Cogito" (Merleau-Ponty, 1993: 381), ya que es consciente que necesita superar el dualismo cartesiano y para eso no basta con invertir la jerarquía de los términos, sino que es necesario buscar una vía o alternativa que la encuentra en la fenomenología (García, 2012: 10). Para Merleau-Ponty, toda la filosofía moderna desemboca en este dualismo cuerpo y espíritu, pero es necesaria una filosofía que parta de cero, que sea capaz desde un inicio de romper con esta tradición de tres siglos, encontrando en la reducción trascendental el método del trabajo y el lenguaje.

Aunque Merleau-Ponty parte de Husserl y fue fiel a su forma reflexiva de pensamiento, efectúa una construcción de una fenomenología de la conciencia comprometida, debido a que el "Ser en el mundo" es un constituyente radical de la existencia humana (Laín, 1988: 379), dando un nuevo sentido a los conceptos de "intencionalidad", "reciprocidad corporal", "sedimentación", "reducción", "horizonte" y el "mundo de la vida", que serán retomados posteriormente por la antropología (Morris, 2015: 351), etnología y etnografía.

Francia se queda sin un referente de la fenomenología tras la muerte prematura de Merleau-Ponty en 1961(San Martín, 1994: 74). No obstante, su legado a la antropología y la disciplina etnográfica en la segunda mitad del siglo XX será entregar una perspectiva del conocimiento humano y la ontología del ser que rompe con el dualismo cartesiano. Las siguientes generaciones no recurrirán a las obras de Edmund Husserl para acercarse a la fenomenología, sino que leerán a MerleauPonty y Alfred Schütz, siendo estas las puertas de entrada para algunos antropólogos, etnógrafos o referentes de las ciencias sociales. Por ejemplo, Pierre Bourdieu,

“Puso en conversación la fenomenología y la hermenéutica heideggeriana con el posestructuralismo, la etnología y el pensamiento de Merleau-Ponty, esto rindió frutos en su ya clásica teoría de la práctica, de los campos y de los capitales" (Wright y Ceriani, 2007: 326).

A su vez, Thomas Csordas retoma la obra de Bourdieu y Alfred Hallowell, prolongando la fenomenología de MerleauPonty (Desjarlais y Throop, 2001: 97) y haciéndola etnográficamente aplicable al concepto de "embodiment", consistente en tomar en cuenta el cuerpo vivido como punto de inicio metodológico (Wright y Ceriani, 2007: 327). Dicho por el propio Csordas, el "embodiment" es "una base común para el reconocimiento de la humanidad del otro y la inmediatez de la intersubjetividad"7 (Katz y Csordas, 2003: 278). En una etnogra-

\footnotetext{
${ }^{7}$ Traducción propia de “...as the common ground for recognition of the other's humanity and the immediacy of intersubjectivity".
} 
fía realizada con Diana Wilson sobre la eficacia terapéutica en la curación ritual navajo, Csordas indaga en la noción de doble horizonte -espacio exterior/espacio corporal- de MerleauPonty (Katz y Csordas, 2003: 277), que consiste en cómo mi cuerpo toma posesión del tiempo, haciendo existir un pasado y un futuro para un presente (Merleau-Ponty, 1993: 254). Otro etnógrafo que reconoce la influencia de la fenomenología de Merleau-Ponty es Clifford Geertz. En una entrevista, publicada en la revista Alteridades, admite una influencia del francés y la fenomenología de Alfred Schütz, en especial al principio de su carrera, aunque no se autoreconozca como un fenomenólogo (Hirsch y Wright, 1993: 124).

La influencia de la fenomenología de Merleau-Ponty es difícil de apreciar, y muchas veces no llega de forma directa a los autores etnográficos. Como ya hemos visto, pensadores como Bourdieu y Geertz, referentes de cómo hacer etnografía, construyeron parte de su pensamiento con la continuación de la fenomenología husserliana realizada por Merleau-Ponty. Ejemplo de esta influencia difusa y oculta es identificada por Matthew Carlin en las etnografías sobre Latinoamérica desarrollada por Michael Taussig (Carlin, 2014: 35). Para Carlin, la influencia de Merleau-Ponty existe de forma anónima en la etnografía de Taussig, estableciendo conexiones en ambos argumentos; explicaciones como que la experiencia precede a la representación, y la posibilidad de la creación de una ciencia atada a lo concreto del mundo y utilizar el arte como intención de ampliar la posibilidad de una experiencia prerreflexiva que se encuentra oculta detrás de nuestra forma de existencia alienada y demasiado cognitiva (Carlin, 2014: 36-38).

Otro autor importante para el desarrollo etnográfico que toma elementos de la fenomenología, en especial de pensadores como Levinas y Merleau-Ponty, es el etnógrafo Michael Jackson, quien enfatizó la inseparabilidad del concepto y la actividad del cuerpo (Mills y Morton, 2013: 101). Jackson toma la idea de Merleau-Ponty de que la intersubjetividad es una modalidad de la vida corporal, donde el significado de la actividad corporal está en la acción misma y no es dado por un agente externo (Jackson, 1996: 32).

2.3. La fenomenología del "mundo de la vida" de Alfred Schütz como referente epistemológico de la disciplina etnográfica.

Pasemos a revisar el pensamiento filosófico de Alfred Schütz. En su obra se observa influencia de varios pensadores, tales como Scheler, Hegel, Bergson, Dilthey (Leal, 2007: 215) y Weber (Leal, 2007: 219). No obstante, encontró en la fenomenología de Husserl los principales recursos metodológicos y teóricos para dar solución a sus preocupaciones de investigación (Leal, 2007: 215) (Overgard y Zahavi, 2009: 3) (Waldenfels, 1997: 91), en especial al estudiar Fenomenología de la Conciencia del Tiempo Inmanente (1928), Lógica Formal y Lógica
Trascendental (1929), Ideas I (1913) y Meditaciones Cartesianas (1931) (Dreher, 2012: 75).

En la obra Fenomenología del mundo social. Introducción a la sociología comprensiva (1932) Schütz comienza a utilizar en sus reflexiones la filosofía fenomenológica, profundizando en sus investigaciones la idea de intencionalidad de la conciencia, la noción de intersubjetividad y de "vida cotidiana" (Leal, 2007: 216). Reconoce que en "la fenomenología trascendental de Husserl se ha establecido un fundamento suficientemente profundo sobre cuya base puede aspirarse a resolver el problema del significado" (Schütz, 1972: 26). Schütz, al igual que Merleau-Ponty, rechaza de manera tajante los programas reduccionistas, conductistas y positivistas que profesan reducir la acción humana solo a un comportamiento observable (Overgard y Zahavi, 2009: 8-9). Schütz nos dice al respecto:

"Un sistema conductista idealmente perfeccionado y totalmente elaborado, por ejemplo, nos alejaría mucho de las construcciones en cuyos términos los hombres experimentan su propia conducta y la de sus semejantes en la realidad de la vida cotidiana" (Schütz, 1974: 38).

Para superar esta dificultad, Schütz identifica la necesidad de contar con recursos metodológicos específicos que permitan considerar pautas de acción racional (Schütz, 1974).

En la estructura conceptual que utiliza Schütz y su relación con la filosofía fenomenológica, formuló una versión modificada del concepto "mundo de la vida" de Husserl, ampliando esta noción al mundo social y las esferas de realidad extra-cotidianas, con una tipicidad esencial (Dreher, 2012: 78) (Overgard y Zahavi, 2009: 10). Schütz analiza en el "mundo de la vida cotidiana" que se experimenta según la noción de actitud natural descrita en la fenomenología husserliana. Por lo tanto, significará abocarse al mundo intersubjetivo que ya existe antes de nuestro nacimiento y que está dado a nuestra experiencia e interpretación, y no sobre "el mundo privado del individuo aislado, sino un mundo intersubjetivo, común a todos nosotros, en el cual tenemos un interés no teórico, sino eminentemente práctico" (Schütz, 1974: 198). Es el mundo de la vida cotidiana el objeto de interés para Schütz, y será a través del análisis filosófico y psicológico de la constitución de nuestra experiencia (Schütz, 1974) lo que le permitirá al investigador social conocer el sistema de construcciones de tipicidades (Schütz, 1974: 39).

Para Schütz existe la necesidad de conocer una tipicidad esencial y crítica de la concepción de Husserl del "mundo de la vida", debido a que no concuerda con una ontología del "mundo de la vida" sin clarificar la noción de intersubjetividad (Schütz, 1974), dicho en otras palabras, "la tentativa husserliana de fundar la constitución de la intersubjetividad trascendental a partir de las operaciones de conciencia del ego trascendental, no han tenido éxito" (Schütz, 1968: 314). Por lo tanto, para Schütz la intersubjetividad no es un problema de constitución que se resuelva dentro de la esfera trascendental, sino que es un dato del mundo de la vida que está fundado sobre la experiencia originaria de la relación en el modo "nosotros" (Schütz, 1968). Según Waldenfels, Schütz critica el supuesto trascendental de Husserl, sustituyendo la subjetividad 
trascendental por una intersubjetividad mundana que presupone de antemano un mundo de la vida social, salvaguardando un lugar a la perspectiva fenomenológica dentro de la investigación social (Waldenfels, 1997: 92).

Volvamos a la transformación o giro que le da Schütz a la noción de intersubjetividad. Esta emerge de manera prereflexiva, como una red de significados que puede ser descubierta y reconstituida por el científico social (Toledo, 2009: 73). Además, amplía sus dimensiones no solo al observador y lo observado, o al yo y tú, sino también a múltiples direcciones del ámbito social, grupal y comunitario mediante el análisis de las condiciones estructurantes de la sociedad (Toledo, 2009). De esta forma, el análisis de las estructuras del mundo de la vida se consolida como el objeto de una ciencia fenomenológica entendida, desde ahora, como una ciencia social (Toledo, 2009). Para Schütz, la estructuración del mundo de la vida, que tiene a su base una intersubjetividad mundana, se da mediante una estratificación que cada uno de nosotros vamos experimentando en el entorno social, mediante "estratos" o "capas" alrededor de sí mismos (Overgard y Zahavi, 2009: 9). Se van estableciendo distintas dimensiones para esta estratificación del mundo de la vida, como son la espacial, temporal y social, dividida en realidades múltiples o ámbitos finitos de sentido (Dreher, 2012: 79).

La teoría schütziana del mundo de la vida influenció a una nueva sociología del conocimiento, permitiendo el desarrollo de una sólida disciplina de investigación empírica cualitativa que impactó directamente en los enfoques etnográficos en los últimos cincuenta años (Dreher, 2012: 105). Es así como el impacto de las reflexiones epistemológicas de Schütz influye en la creación de propuestas metodológicas cualitativas y teóricas como son la etnometodología de Harold Garfinkel, la teoría fundada de Glasser y Strauss, la propuesta de Erving Goffman del frame analysis, la hermenéutica social científica propuesta por Soeffner, y el enfoque cualitativo propuesto por Peter Berger y Thomas Luckmann en la “Construcción Social de la Realidad".

Profundizaremos ahora en la etnometodología de Harold Garfinkel, y el enfoque cualitativo propuesto por Peter Berger y Thomas Luckmann en la "Construcción Social de la Realidad", donde es posible apreciar parte de la propuesta socio-fenomenológica de Schütz y las influencias de segunda mano de Husserl, pero no una articulación de los constructos de ambos autores, y menos aún, una extensión de la propuesta fenomenológica. Comencemos por revisar brevemente la propuesta etnográfica de Harold Garfinkel, quien publica en 1967 su libro Estudios en Etnometodología, proponiendo una metodológica cualitativa conocida en la actualidad como "Etnometodología". Garfinkel toma la idea básica de Schütz de que "los tipos ideales son construcciones teóricas con funciones eminentemente heurísticas que tienen que adecuarse empíricamente a los motivos y razonamientos que tuvo el actor en la situación original" (Velasco, 2012: 219), buscando a través de este método mostrar la racionalidad de la acción en relación a los conocimientos, intenciones y valores de los actores sociales, dejando de lado los conocimientos del intérprete (Velasco, 2012). En la búsqueda de documentar el carácter empírico del conocimiento y razonamiento del sentido común, explotará el marco conceptual del Alfred Schütz, en particular, lo concerniente a la teoría del mundo de la vida y la noción de acción social (Firth, 2010: 607). Con respecto al mundo de la vida, utilizará las descripciones de Schütz sobre las actitudes de la vida cotidiana y las actitudes de la teorización científica, fundamentando que ambas actitudes no se solapan y producen conjuntos de eventos lógicamente incompatibles (Garfinkel, 2006: 304-306311). No obstante, Garfinkel realiza una interpretación de las concepciones del mundo de la vida o de las actitudes de la vida cotidiana de Alfred Schütz con "un sentido empírico no doctrinal" (Garfinkel, 2006). Para ilustrar esta idea, en palabras de Garfinkel (2006):

“En vez de tratar las propiedades de la racionalidad como principios metodológicos para interpretar las actividades, éstas deben ser tratadas como material empíricamente problemático. Deben tener el estatus solo de datos y deben ser explicadas de la misma manera en que se explican las propiedades más familiares de la conducta" (Garfinkel, 2006: 317).

Posterior a la década de los sesenta, Garfinkel se convirtió en una Personaje que influenció a una serie de figuras antropológicas y etnográficas de universidades norteamericanas, tan diferentes en sus propuestas como lo son Bennetta JulesRosette, Johannes Fabian, George Psathas, Gerald Berreman, Michael Agar y Lawrence Wieder (Reynoso, 1998: 146). Otros autores influenciados por Alfred Schütz y con un gran impacto en la teoría del conocimiento de la antropología y disciplinas etnográficas son: Peter Berger y Thomas Luckmann.

Es por medio de la obra La Construcción Social de la Realidad que sus discípulos Thomas Luckmann y Peter Berger, desarrollan una posición contraria al estructural funcionalismo de Talcott Parsons, estableciendo un cambio determinante en la teorización e investigación sociológica (Dreher, 2012: 92). Luckmann continúa el proyecto de Schütz proponiendo una protosociología, cuya intención consiste en diferenciar entre las metodologías de la fenomenología y la sociología, estableciendo una acción conjunta y paralela para dar explicación (Dreher, 2012: 103-107).

El objeto al que se refieren es el de la sociedad como parte de un mundo humano, un mundo compuesto y habitados por personas con una historia en común. Así, presenta una teoría del mundo de la vida basada en la fenomenología de Alfred Schütz (Rizo, 2015: 25) donde, a partir de las experiencias individuales se describen las estructuras del mundo de la vida cotidiana, ajustando de lo individual a lo social, de lo natural a lo histórico, y de lo originario a lo cotidiano, argumentando que la realidad se construye socialmente (Rizo, 2015: 24), e instaurando una investigación paralela con bases en la fenomenología y la sociología.

¿Cómo establece Luckmann una acción paralela de la investigación fenomenológica y sociológica? Lo hace confrontando las descripciones fenomenológicas a través de análisis constitutivos de la conciencia subjetiva y análisis sociológicos empíricos en diferentes ámbitos de investigación como el mundo social, las acciones comunicativas, el ritual y el símbolo, entre otros elementos (Dreher, 2012: 93). Por ejemplo, en su 
investigación sobre los límites del mundo social analiza, por una parte, la constitución fenomenológica del mundo humano y social, y, por otra, los límites empíricos del mundo social a partir de los resultados de estudios antropológicos (Dreher, 2012). El ajuste entre estas dos líneas de investigación paralela -sociológicos y antropológicos- las realiza entorno a la antropología, materializándose en un avance de la ciencia social con trasfondo empírico-cualitativa. Lo anterior se puede deber a que Luckmann realiza una crítica a la fenomenología de Husserl que se traduce, en palabras de Jochen Dreher, en la noción de ego trascendental de Husserl, aunque constituye el mundo entero, incluyendo al alter ego que mantiene oculta su cualidad humana (Dreher, 2012: 95). Aun así, no deja de utilizar ideas fenomenológicas como el mundo de la vida, la intencionalidad de la conciencia, paréntesis fenomenológico, intersubjetividad, entre otros (Berger y Luckmann, 2001). En definitiva, la fenomenología del mundo social de Luckmann está mucho más cerca de la sociología que de la fenomenología husserliana, utilizando esta última para restablecer la construcción de la realidad social (Rizo, 2015: 23). Además, podemos constatar que en la búsqueda de conciliar la relación dialéctica entre individuo y sociedad, Luckmann no abandona una investigación empírica del mundo de la vida basada en el dato:

"Solo quisiéramos agregar aquí que, en nuestra opinión, la investigación empírica sobre la relación de las instituciones con los universos simbólicos legitimadores contribuirá grandemente a la comprensión sociológica de la sociedad contemporánea" (Berger y Luckmann, 2001: 231).

Ahora bien, no abandonan una mirada empírica puesto que al realizar una acción paralela de investigación fenomenológica y sociológica, mantienen las descripciones sociológicas empíricas, llevando a igualar el análisis fenomenológico con el método descriptivo y una perspectiva empírico no científica.

Por ejemplo, en sus investigaciones sobre los fenómenos religiosos como el animismo, chamanismo y totemismo, Luckmann utiliza apreciaciones de la antropología y los estudios comparativos, llegando a la conclusión que "lo Humano y lo social no se pueden equiparar bajo ninguna circunstancia; dejando claro, que solo el Yo empírico y mundano puede alcanzar la humanidad." (Dreher, 2012: 95). Esta visión metodológica y teórica de Luckmann que no abandona del todo el empirismo o renuncia a una reducción trascendental fenomenológica como la propuesta por Husserl, obtuvo una gran influencia en la antropología social, en la etnografía y en la teoría del conocimiento de las ciencias sociales. En especial, en lo referente a la construcción social de la realidad, o de un "Construccionismo Social", que concibe el mundo social conformado por patrones o pautas de interacción que están antes de nuestro nacimiento, pero con las que nos involucramos y nos permiten aprender y crear significados (Rizo, 2015: 21-22); similar a la concepción de "mundo de la vida" de Schütz, o de la teoría de la "actitud natural" de Husserl, con otro trasfondo y menor densidad. Por lo tanto, el núcleo de la "teoría social de la realidad" de Luckmann y Berger, se centra en que los sujetos crean la sociedad y esta es en una realidad de carácter objetivo (Rizo, 2015: 23). Comparten con Schütz una noción de la intersubje- tividad que se basa en una comunidad social e histórica, abandonando la noción que se centra en el flujo de conciencia interior. También es importante mencionar a Clifford Geertz, uno de los primeros antropólogos y etnógrafos que aplicó ideas fenomenológicas usando una discusión inspirada en Schütz, y distinguiendo entre las orientaciones del sentido común, científico, estético y religioso (Desjarlais y Throop, 2011: 89).

Las influencias epistemológicas del pensamiento fenomenológico de Schütz y Merleau-Ponty no se agotan en los autores de las ciencias sociales que hemos revisado, debido a que variadas influencias de la fenomenología de Husserl impactaron de forma difusa en otras corrientes de pensamiento, estableciendo un parentesco con la hermenéutica de Paul Ricoeur (Ricoeur, 2000), el horizonte hermenéutico de Hans Georg Gadamer (Reynoso, 2015), la noción de "quiebre" de Michael Agar, entre otros autores. Aunque los autores de las ciencias sociales que hemos estudiado por su directa influencia de la fenomenología de Alfred Schütz no adhieren a una concepción empirista dogmática del conocimiento, sí establecen un desarrollo metodológico empírico cualitativo en sus concepciones de lo que debe ser la disciplina etnográfica. Esto puede tener un origen en la metodología schütziana del mundo de la vida, la que resulta influyente en los procesos de construcción de los métodos de las ciencias sociales empírico cualitativas (Dreher, 2012: 91), a diferencia de un Merleau-Ponty donde el devenir de su pensamiento llega al extremo de crear un nuevo lenguaje en su obra póstuma Lo visible y lo invisible para evitar caer en cualquier tipo de empirismo al acercarse a un "otro".

3. El abandono de la fenomenología de Husserl: ¿Por qué se abandona la noción de intersubjetividad como un a priori del conocimiento?

Si nos centramos en los puentes establecidos entre la filosofía y las ciencias sociales por Merleau-Ponty y Schütz, que influyeron a la etnografía en algunos casos de forma directa y en otros indirectamente, e incluso de manera soterrada con las ideas de la fenomenología husserliana, ¿por qué esta última no continuó con una influencia más profunda en las ciencias sociales y en la etnografías de las décadas pasadas? O, dicho de otra forma, si el constructo teórico de la intersubjetividad tiene su origen en la fenomenología de Husserl, ¿por qué fue abandonada su crítica, análisis y reformulación? Esta pregunta presenta varias complejidades que no permiten establecer una respuesta concluyente debido a que con la irrupción de un posmodernismo en las ciencias sociales se establece un descrédito a todo lo que busque un fundamento en la metafísica (Reynoso, 1998: 126), criticando fuertemente a la modernidad y todo pensamiento del que amerite una sospecha, lo que se tradujo en una fragmentación de los grandes metarelatos. Sumado a lo anterior, la irrupción en los años sesenta del postestructuralismo y su crítica, procesamiento y radicalización de 
las ideas estructuralistas por sus principales protagonistas Jacques Derrida, Michel Foucault, Jacques Lacan, Jean-Luc Nancy, Gilles Deleuze, Judith Butler y Ernesto Laclau tendrán una importancia creciente en las sociologías y ciencias de la cultura (Moebius, 2012: 495), dificultando establecer relaciones de influencia con las ideas filosóficas anteriores.

No obstante, podemos acercarnos a conocer cómo fue recibida la fenomenología de Husserl, Schütz, Merleau-Ponty, no solo por quienes toman ciertos elementos de esta filosofía y los llevan a las ciencias sociales, sino también por otro grupo de pensadores que la negaron y criticaron de forma radical, viendo en ella una antítesis de sus ideas.

En dos obras comúnmente citadas por antropólogos, nos referimos a Esquisse d'une théorie de la pratique (1972) y Le sens pratique (1980). Pierre Bourdieu realiza una crítica a la fenomenología, incluyendo a "la filosofía existencial de Sartre, la Etnometodología de Garfinkel, la fenomenología social de Schütz y la fenomenología genética de Husserl" (Throop y Murphy, 2002: 186-189), agrupándolas a todas de forma genérica en una crítica amplia del subjetivismo (Throop y Murphy, 2002: 187).

El primero de ellos es Alfred Schütz, quien encarna la visión subjetivista (Dreher, 2012: 132) caracterizada por apoyarse demasiado en una acción intencionada de carácter premeditado, continuando una falacia intelectualista de las ciencias de la razón (Throop y Murphy, 2002: 195). En este caso, Bourdieu realiza una mala interpretación de la posición de Schütz frente a la modelación de la acción social, quien no solo deja espacio para una acción pre-reflexiva dirigida por un sentido práctico, también demuestra que los motivos y proyectos conscientes desempeñan un importante papel en la modelación de la acción social (Throop y Murphy, 2002). Otra crítica a la fenomenología la dirige contra la Etnometodología de Garfinkel, por considerar que promueve una ilusión ocasional, ya que no es capaz de ver que las relaciones interpersonales no se restringen nunca a las interacciones individuales de los individuos, debido a que siempre hay restricciones estructurales que son más amplias (Throop y Murphy, 2002: 190). A Jean Paul Sartre lo acusa de un "ultra-subjetivismo", que se expresa en una conciencia sin inercia que va creando el sentido de un mundo nuevo en cada momento, y que solo puede encontrar continuidad en la fidelidad así mismo (Bourdieu, 1990: 46). Al igual que con Schütz, Bourdieu (1990) critica a Sartre por un intelectualismo en sus ideas, expresadas en un subjetivismo de carácter universal:

"Comprometido con la ilusión de «conciencia sin inercia», sin pasado y sin exterior, dota a todos los sujetos con los que decide identificar, es decir, casi exclusivamente el "pueblo» proyectivo nacido de esta "generosa" identificación con su propia experiencia como un sujeto puro y flotante" (Bourdieu, 1990: 46).

En un artículo del 2002 publicado en la revista Anthropological Theory, Jason Throop y Keith Murphy elaboran una serie de fundamentaciones llegando a concluir y argumentar que las críticas realizadas por Bourdieu al subjetivismo y la fenomenología se centran en una caracterización errónea del esfuerzo fenomenológico y, al mismo tiempo, no es capaz de reconocer que su proyecto se superpone y se basa en las perspectivas fenomenológicas de Husserl y Schütz (Throop y Murphy, 2002: 191). Es interesante cómo justifican una analogía o relación entre la conceptualización de "Doxa" realizada por Bourdieu, y la noción de "actitud natural" presente en el devenir del pensamiento de Husserl en sus últimas obras, esbozando las bases de una fenomenología genética que explique cómo el mundo de la vida "doxica" se establece y mantiene a través de la conciencia que se moldea por medio de la sedimentación de la vida pasada (Throop y Murphy, 2002). Además, afirman que Bourdieu ha minimizado el uso de la versión del propio Husserl del concepto de "habitus", existiendo una superposición no reconocidas por el autor galo entre ambas versiones. Meses antes de su muerte, Pierre Bourdieu responde a los emplazamientos de Throop y Murphy en una nota enviada a la misma revista, donde reconoce una influencia fenomenológica en su juventud y que su finalidad es integrar el análisis fenomenológico en un enfoque global que no pretende ser una compilación ecléctica, solo conservar las contribuciones esenciales (Bourdieu, 2002: 209). Además, enfatiza que:

“La mala interpretación de mis ideas está enraizada en el hecho de que los autores se olvidan de que, mi intención, las ideas teóricas que tratan aisladamente, por separado, en y para sí, están diseñadas para guiar la investigación empírica y para resolver problemas específicos de la antropología y la sociología, como el problema del intercambio de obsequios o el del trabajo, por lo que propuse, en las meditaciones pascalianas, un análisis integrador de las visiones subjetivista y objetivista, así como de muchos otros a lo largo de mi carrera investigadora" (Bourdieu, 2002: 209) .

Otra poderosa y particular crítica realizada contra la fenomenología proviene del postestructuralismo de Jacques Derrida. Su pensamiento está inserto en un conjunto de diferentes concepciones teóricas desarrolladas en la década del sesenta en Francia, que mantienen los supuestos fundamentales de las teorías del lenguaje de las corrientes estructuralistas, realizando un procesamiento y radicalización de este pensamiento, con protagonistas ilustres como son Michel Foucault, Jacques Lacan, Jean-Luc Nancy, Gilles Deleuze, Judith Butler y Ernesto Laclau (Moebius, 2012: 495). Jacques Derrida extiende su ataque a toda forma de fundamentación que se base en la metafísica occidental (Leyva, 2012: 195), al igual que las ciencias sociales postestructuralistas que critican una "metafísica de la presencia" que se encuentra en una serie de metáforas descriptivas con apariencia analítica (Moebius, 2012: 500). Para Derrida, la fenomenología husserliana, del mismo modo que la metafísica, olvidaría la diferencia entre el origen del sentido (su acontecimiento) y el sentido puro (la significación). En otras palabras, Husserl intenta definir el querer-decir último del sentido con la noción de vivencia, y pensando la fenomenología como la Ciencia de esas vivencias, pensaría agarrar el origen (la vivencia en cuanto relación eidética), olvidando cierto retardo del origen (el diferir constitutivo y sin descanso del origen desde el sentido) (Potestà, 2013: 176). 
Lo que intentará hacer Derrida es romper con la tradición de la metafísica, donde la fenomenología trascendental de Husserl sería su momento último debido a una comprensión problemática de la "voz" (Potestà, 2013: 173-174). Esta "voz" en la metafísica y la fenomenología correspondería a la presunción problemática de "una presencia pura del sentido de una conciencia" (Potestà, 2013: 174-188). Por lo tanto, en el caso de la fenomenología trascendental, siempre la "voz", que está marcada por la intencionalidad, tendrá preponderancia por sobre la "escritura", que se mantiene relegada en segundo lugar, al igual que el "signo" frente al "significante" (Potestà, 2013: 188).

Aunque la crítica que realiza Derrida utiliza argumentos que demuestran un conocimiento profundo de las primeras obras de Husserl, no necesariamente será así con los seguidores de las ideas del postestructuralista en las ciencias sociales en la década del sesenta y posteriores. Principalmente, estarán focalizadas en relacionar el pensamiento fenomenológico con una metafísica clásica que se caracteriza por propiciar ciencias sociales que establecen postulados ahistoricos y universales. Por lo tanto, no implicará un conocimiento directo de Husserl, sino más bien, un acercamiento a la fenomenología de carácter fragmentario, utilizando como vehículo la intención de quebrar la metafísica principalmente con las ideas de Husserl.

Los reproches provenientes de Bourdieu y de los postestructuralistas como Derrida contra el pensamiento estructuralista $^{8}$ son homologadas de forma errónea en contra de la fenomenología desarrollada por Husserl, provocando que la continuidad del pensamiento husserliano, como también la continuidad de la fenomenología realizada por Schütz y Merleau-Ponty, no sea una opción común en la búsqueda de sustento ontológico y epistemológico de las ciencias sociales $y$, en especial, de la etnografía de los años setenta y ochenta. Johannes Fabian grafica muy bien el comienzo como antropólogo y etnógrafo en su libro Memory Against Culture: Arguments and Reminders, donde la influencia de la fenomenología es casi nula en la concepción epistemológica de un "otro" y la construcción teórica de la intersubjetividad en los años setenta (Fabian, 2014: 171).

4. El retorno de las ideas al conocimiento del nosotros y la intersubjetividad

Tal como nos menciona Merleau-Ponty(Merleau-Ponty, 2012), la originalidad de pensamiento de Husserl es que buscó

\footnotetext{
8 Las corrientes estructuralistas establecen la pretensión teórica de constituir el análisis de la ley de actividad simbólica en un fundamento de las ciencias humanas de forma análoga a las ciencias naturales, o de considerar al individuo como subjetivado y construido por la estructura, conduciendo a un enfoque teórico y metodológico marcado por la ahistoricidad y universalidad de sus postulados que trasciende las diferentes culturas, pudiéndose aplicar a cualquier orden simbólico, cultura o grupo humano (Moebius, 2012: 491).
}

un camino intermedio entre el logicismo ${ }^{9}$ y el psicologismo ${ }^{10}$ sin caer en el método deductivo o el conocimiento simplemente empírico. Husserl franquea estos límites (Merleau-Ponty, 2011:26-30-32) y los supera por medio de una filosofía de la intersubjetividad: "La intersubjetividad universal, en la que se resuelve toda objetividad, todo lo que existe, no puede, manifiestamente, ser otra que la humanidad que innegablemente es una parte componente del mundo" (Husserl, 2008: 220).

Por la tanto, si tomamos en consideración el devenir completo del pensamiento de Husserl, incluyendo los manuscritos publicados de forma póstuma, o el denominado "Nuevo Husserl", vemos que las críticas realizadas por ilustres e influyentes pensadores como Bourdieu, Derrida, y otros no tan ilustres ni tan influyentes, establecen una mirada parcial que no reconoce que la fenomenología trascendental aportó una nueva mirada desde la constitución del "otro" y del "nosotros".

Se aprecia en los últimos treinta años que los enfoques fenomenológicos se han hecho cada vez más importantes en la reflexión antropológica y etnográfica sobre un variedad de temáticas: la experiencia vivida, la enfermedad y la curación, el sufrimiento, la violencia, la moralidad, la corporalidad, la percepción sensorial, las prácticas comunicativas, la mente y la conciencia, la creatividad y los esfuerzos estéticos y la subjetividad e intersubjetividad (Desjarlais y Throop, 2011: 88-89-90).

Estos antropólogos y etnógrafos que, a mediados de los ochenta, aplicarán la fenomenología a problemáticas propias de la etnografía, son inspirados por la incursión ocasional de Clifford Geertz, Alfred Irving Hallowell, David Bidney, Pierre Bourdieu, Lawrence Watson, Víctor Turner, entre otros; debido a que consideran que la antropología se había centrado de forma insuficiente en temáticas del significado, discurso, relaciones estructurales y economía política, abandonando las experiencias cotidianas (Desjarlais y Throop, 2011: 92).

Entre los etnógrafos que retoman la filosofía de Husserl destaca el neozelandés Michael Jackson, quien extiende la fenomenología a muchos lugares con varias de sus obras, entre las que destaca su introducción "Phenomenology, radical empiricism and anthropological critique" en el libro "Things as they are: New directions in phenomenological anthropology" (Jackson, 1996) estableciendo no solo una dirección, más bien una multiplicidad fenomenológica de nuevas formas etnográficas de presentar a un otro. Jackson argumenta en sus investigaciones que la intersubjetividad es un concepto clave en la investigación antropológica, llevando al límite su escritura etnográfica (En Australia central con tribus Warlpiri), adoptando una antropología de la experiencia que se basa en Husserl y en otros autores, incluyendo a William James, John Dewey, Martin Heidegger, Hannah Arendt y Maurice Merleau-Ponty (Duranti, 2010: 4). Jackson utiliza los diferentes desarrollos fenomenológicos de estos autores para comprometerse con un proyecto existencialista que extiende los límites del concepto y la práctica de la etnografía (Duranti, 2010), explorando “las formas en

\footnotetext{
${ }^{9}$ Actitud que admite que existe una verdad, más allá de la cadena de las causas y efectos psicológicos y sociales (Merleau-Ponty, 2011: 26).

${ }^{10}$ Conciben la vida del ser humano resultante de condiciones exteriores que actúan sobre él y que ven al sujeto filosofante completamente determinado desde el exterior, sin contacto con su propio pensamiento (Merleau-Ponty, 2011: 30)
} 
que una serie de compromisos intersubjetivos, desde el juego y los fetiches, hasta la violencia y la religiosidad, implican imbricaciones complejas del sujeto y el objeto, el yo y el otro" ${ }^{11}$ (Desjarlais y Throop, 2011: 89).

A finales de los ochenta y principios de los noventa, Thomas Csordas ${ }^{12}$ retoma la obra de Alfred Irving Hallowell, la fenomenología del cuerpo de Merleau-Ponty y el concepto de "habitus" de Bourdieu como práctica no reconocida, desarrollando el concepto de "embodiment", "el cual implica tomar en cuenta el cuerpo vivido como punto de partida metodológico antes que como objeto de estudio" (Wright, 2007: 327). Para Csordas el cuerpo es el punto de partida para analizar la cultura y el "yo", buscando superar la dualidad objeto y sujeto mediante una reflexión que se centra en la percepción (lo pre-objetivo) y la práctica (el habitus), fundamentado en el cuerpo (Csordas, 1990: 39). Será a través de la noción de "embodiment" (comprensión mutua) que es posible realizar una serie de preguntas sobre la experiencia y la percepción religiosa (Csordas, 1990: 40).

Otro antropólogo que ha realizado una aplicación de las ideas de Husserl en sus investigaciones etnográficas del ámbito del lenguaje es Alessandro Duranti. Visibiliza la relevancia de la filosofía de Husserl en la comprensión del papel que cumple el lenguaje en la socialización de la atención, mediante la aplicación de la teoría de la "actitud natural" de Husserl (Duranti, 2009: 205). Propone releer el uso del concepto de Husserl de "natural" como un correspondiente del concepto de cultura (Duranti, 2009). Tal como revisamos anteriormente, Duranti propone reexaminar el concepto de intersubjetividad desde la mirada de Husserl, identificando más de seis dominios o aplicabilidades del concepto presentes en la fenomenología husserliana, a diferencia de la interpretación restringida que existe hoy en día como "comprensión mutua o compartida"13 de Alfred Schütz (Duranti, 2010: 4).

Duranti es consciente de que el concepto de intersubjetividad para Husserl es la categoría ontológica fundamental de la existencia humana, y que no es un producto o un efecto de la comunicación, es una condición para su posibilidad (Duranti, 2010: 9). Uno de sus últimos libros, The Anthropology of Intentions, realiza una cuidadosa recopilación de tres décadas de trabajo etnográfico en grupos humanos de Estados Unidos y el Pacífico, demostrando desde una perspectiva etnográfica que las intenciones entendidas como disposiciones cognitivas, emocionales y corporales, siempre están incrustadas en un mundo intersubjetivo de la experiencia, utilizando para este análisis la concepción de Husserl de la "intencionalidad" y la "intersubjetividad". (Duranti, 2015: 1-2).

Jason Throop, antropólogo y etnógrafo que ha reivindicado y aplicado la filosofía fenomenológica en el trabajo etnográfico, en los campos de la antropología médica y psicológica, estableció un compromiso en el ámbito de las exploraciones de patrones culturales del dolor, y basa su trabajo en Edmund Husserl y Emmanuel Levinas para concluir cómo las

\footnotetext{
${ }^{11}$ Traducción propia.

12 Indirectamente a través de Schütz, Csordas está retomando la noción de "corporificación" de Husserl (Husserl, 1997: 130).

13 Duranti utiliza la expresión "shared or mutual understanding" (Duranti, 2010: 4).
}

experiencias del dolor de habitantes de las islas Yap (Micronesia) permiten una comprensión de la experiencia moral que va más allá de las representaciones tradicionalmente ofrecidas (Throop, 2010: 16). También reflexiona sobre los problemas de la experiencia en la teoría antropológica contemporánea, haciendo una llamada a los antropólogos y etnógrafos a repensar los beneficios potenciales ofrecidos por la fenomenología de Husserl y Schütz para la investigación antropológica (Throop y Murphy, 2002: 186). Este, llamado a realizar una revisión a la fenomenología, lo hace en torno de los constructos teóricos de Pierre Bourdieu, como son el de "Doxa"y "Habitus", que se solaparían con las ideas de la fenomenología de Husserl (Throop y Murphy, 2002: 191), cuando introduce el término "protodoxa" (urdoxa) o "creencia primitiva" al expresar "la referencia intencional retrospectiva de todas las $<$ modalidades de creencia $>$, puesta de manifiesto por nosotros" (Husserl, 1997b: 252).

Tim Ingold, es otro etnógrafo fenomenológico ${ }^{14}$, que establece una directa influencia de Merleau-Ponty en el giro hacia el espacio, cuerpo y la noción de encarnación (Downey, 2015: 118). Para Ingold los seres humanos no están separados de lo que pasa en el entorno o paisaje, las respectivas materialidades interfieren en el transcurso de los acontecimientos (Ingold, 2011:29) y va más allá del "análisis existencial" de la reflexión fenomenológica sobre el cuerpo, o "la carne" en un espacio intersubjetivo (Merleau-Ponty, 1993: 153), ya que para Ingold los cuerpos se convierten en organismos igual que otras materialidades sin distinción y "participan desde dentro en su ulterior transformación"15 (Ingold, 2011: 29). Los aportes de Ingold han sido re-localizados hasta la arqueología del paisaje (Ingold, 1993), estableciendo una compresión intersubjetiva, ya no solo en los "otros", sino también con y entre los objetos del paisaje.

Existen otros ejemplos de investigadores de las ciencias antropológicas que han realizado un puente entre la etnografía y la filosofía fenomenológica en los últimos treinta años. Víctor Crapanzano resalta los sesgos lingüísticos y culturales de los escritos fenomenológicos (Desjarlais y Throop, 2011) ampliando la noción de "Horizontes" a la de "Horizontes imaginativos" (Crapanzano, 2004: 20). Byron Good busca avanzar en una fenomenología crítica en el ámbito de la antropología médica (Csordas, 1990: 96), y desde la vereda de la filosofía, Javier San Martín ha intentado acercar la fenomenología husserliana a los estudios propios de la antropología en las temáticas de la interculturalidad y la intersubjetividad (San Martín, 2015). Los aportes anteriores han intentado no solo adoptar una serie de constructos teóricos como la teoría de la "actitud natural", la "intencionalidad", la "epojé" y la "intersubjetividad", sino que también se han propuesto reflexionar críticamente sobre los límites de la fenomenología, buscando ampliar su alcance y aplicabilidad $^{16}$ (Desjarlais y Throop, 2011: 97).

\footnotetext{
14 Aunque Ingold se autodenomina "I am an anthropologist: not a social or cultural anthropologist; not a biological or archaeological anthropologist; just an anthropologist" (Ingold, 2011).

15 Traducción propia: "And in this respect human beings are no exception. They are, in the first place, organisms, not blobs of solid matter with an added whiff of mentality or agency to liven them up. As such, they are born and grow within the current of materials, and participate from within in their further transformation".

16 Desjarlais y Throop se refieren a los aportes de Crapanzano, Csordas, Jackson, Duranti.
} 


\section{Conclusiones}

Husserl llegó tarde a la tarea de establecer una articulación entre fenomenología y antropología, tomando en consideración la cantidad de tiempo que dedicó para desentrañar la relación entre fenomenología y la psicología (Zirión, 2015: 211). Nos relata entre líneas la necesidad de las investigaciones etnológicas y antropológicas de orientar toda investigación a las claves fenomenológicas (Zirión, 2015), y de la misma forma que hemos visto la incorporación de estas claves fenomenológicas presentes en Husserl, Merleau-Ponty y Schütz, también lo hemos visto en antropólogos y etnógrafos que en las últimas décadas han aplicado, reflexionado y criticado estas ideas en sus investigaciones etnográficas ${ }^{17}$.

En el caso de las ciencias sociales en general, no aplica la excepción antes vista; la influencia de Husserl ha sido mayoritariamente indirecta a través de sus discípulos filósofos. Nosotros nos hemos focalizado en Alfred Schütz y Merleau-Ponty, por su influjo más directo en la antropología y la etnografía, posibilitando que las ideas de Husserl permanecieran muchas veces de forma soterrada, no reconocida, o simplemente no identificada, en una parte de las corrientes de pensamiento que han marcado a la antropología en los últimos cincuenta años $^{18}$.

A pesar de esta influencia indirecta, o el rechazo que ha provocado en algunos autores, no podemos dejar de reconocer que la fenomenología husserliana ha proporcionado los argumentos para un posicionamiento de las teorías del individuo o de agencia, permitiendo un equilibrio con las posturas teóricas que condicionan el actuar de las personas en la estructura social. No obstante, la intención de Husserl era encontrar un camino intermedio y no una oposición.

Para finalizar, mencionar que aunque esta tercera generación de etnógrafos y antropólogos (Csordas, Jackson, Duranti, Throop, Ingold, Good, San Martín, Crapanzano) tienen miradas diferenciadas en torno al énfasis y desarrollo del pensamiento fenomenológico aplicado al trabajo etnográfico, convergen en un punto: la intersubjetividad como terreno en común de la investigación de un "otro", o tal vez de un "nosotros", para darle mayor sustento epistemológico, profundidad y diversidad a la disciplina etnográfica.

\footnotetext{
17 Crapanzano, Csordas, Desjarlais, Good, Throop, Jackson, Duranti.

18 Geertz, Bourdieu, Garfinkel, Natanson, Luckmann, Berger, por nombrar algunos.
} 
Bibliografía

BERGER, P. y Luckmann, T. (2001): La construcción Social de la realidad. Buenos Aires, Amorrortu Editores.

BIEMEL, W. (1968): Husserl, Tercer coloquio filosófico de Royaumont. Buenos Aires, Paidós.

BOURDIEU, P. (1990): The Logic of Practice. California, Stanford University Press.

BOURDIEU, P. (2002): "Response to Throop and Murphy", Anthropological Theory, 2 (2), p. 209.

BRUNER, J. (1996): The Culture of Education. Cambridge, MA, Harvard University Press.

CARLIN, M. (2014): “El fetiche etnográfico de Michael Taussig", EntreDiversidades Revista de Ciencias Sociales y Humanidades, 2, pp. 13-44.

CSORDAS, T. J. (1990): "Embodiment as a Paradigm for Anthropology", Revista Ethos, 18(1), pp. 5-47.

KATZ, J. y CSORDAS, T. J. (2003): “Phenomenological ethnography in sociology and anthropology", Ethnography, 4 (3), pp. 275-288.

DESJARLAIS, R. y Throop J. C. (2011): "Phenomenological approaches in anthropology", Annual review of anthropology, 40, pp. 87-102.

DREHER, J. (2012): “Fenomenología: Alfred Schütz y Thomas Luckmann”, en E. de la Garza y G. LEYVA (eds.), Tratado de metodología de las ciencias sociales: perspectivas actuales, México: Fondo de Cultura Económica, pp. 97-139

DURANTI, A. (2009): "The relevance of Husserl's theory to language socialization", Journal of Linguistic Anthropology, 19 (2), pp. 205-226.

DURANTI, A. (2010): "Husserl, intersubjectivity and anthropology", Anthropological Theory, SAGE Publication Vol 10 (1), pp. 1-20.

DURANTI, A. (2015): The anthropology of intentions. Inglaterra, Cambridge University Press.

FABIAN, J. (2014): Time and the other: How anthropology makes its object. Nueva York, Columbia University Press.

FIRTH, A. (2010): "Etnometodología", Revista Discurso y Sociedad, Traducido por Teresa E. Cadavid G., 4 (3), pp. 597-614.

GARCÍA, E. A. (2012): Maurice Merleau-Ponty: filosofía, corporalidad y percepción. Buenos Aires, Editorial Rhesis.

GARFINKEL, H. (2006): Estudios en Etnometodología. Barcelona, Anthropos.

GEERTZ, C. (1991): "Desde el punto de vista de los nativos: sobre la naturaleza del conocimiento antropológico", Alteridades, 1 (1), pp 102-110.

GUBER, R. (2001): La Etnografía; Método, Campo y Reflexividad. Bogotá, Grupo Editorial Norma.

HABERMAS, J. (1970): "Towards a theory of communicative competence", Inquiry, 13 (1-4), pp. 360-375.

HIRSCH, S. M., y WRIGHT, P. G. (1993): “De Bali al posmodernismo: una entrevista con Clifford Geertz", Alteridades, 3 (5), pp. 119-126.

HUSSERL, E. (1972): “La pregunta por el origen de la geometría como problema histórico-intencional". Traducción de Raúl Velozo Farias, Documentos. Instituto de filosofía (universidad de Chile). Translation of Husserliana VI, pp.365-386 (Beilage III), pp. 16-78.

HUSSERL, E. (1984): Crisis de las Ciencias Europeas y la Fenomenología Trascendental. México, Editorial Folio Ediciones.

HUSSERL, E. (1997): Filosofía contemporánea. Ideas relativas a una fenomenología pura ya una filosofía fenomenológica. Libro segundo. Investigaciones Fenomenológicas Sobre la constitución. México, Editorial Universidad Nacional Autónoma de México.

HUSSERL, E. (2013): Ideas relativas a una fenomenología pura y una filosofía fenomenología, Libro primero: Introducción general a la fenomenología pura. México, Fondo de Cultura Económica.
INGOLD, T. (1993): “The temporality of the landscape”, World archaeology, 25 (2), pp. 152-174.

INGOLD, T. (2011): Being alive: Essays on movement, knowledge and description. Londres, Routledge .

JACKSON, M. (ed.) (1996): Things as they are: New directions in phenomenological anthropology. Georgetown University Press.

LAÍN, P. (1988): Teoría y realidad del otro. Madrid, Alianza.

LEAL, R. (2007): "Aportes teórico-metodológicos de la fenomenología al desarrollo cualitativo de las ciencias sociales en Alfred Schutz", Alpha. I, 25, pp. $215-225$.

LEYVA, G. (2012): “La Hermenéutica clásica y su impacto en la epistemología y teoría social hoy", en E. de la Garza y G. Leyva (eds.), Tratado de metodología de las ciencias sociales: perspectivas actuales. México, Fondo de Cultura Económica, pp. 432-465.

LÓPEZ, M. (1996): “La fenomenología existencial de M. Merleau-Ponty y la sociología", Papers: revista de sociologia, 50, pp. 209-231.

LÓPEZ, M. (2004): "Intersubjetividad como Intercorporeidad", La Lámpara de Diógenes. 5, pp. 57-70.

MERLEAU-PONTY, M. (1993): Fenomenología de la Percepción. Barcelona, Planeta de Agostini.

MERLEAU-PONTY, M. (2011): La Fenomenología y las Ciencias Humanas. Buenos Aires, Prometeo Libros.

MILLS, D., y MORTON, M. (2013): Ethnography in education. Londres, Sage.

MOEBIUS, S. (2012): "Postestructuralismo y ciencias sociales”, en E. de la Garza y G. Leyva (eds.), Tratado de metodología de las ciencias sociales: perspectivas actuales. México, Fondo de Cultura Económica, pp. 488-533.

MORAN, D. (2012): Husserl's Crisis of the European Sciences and Transcendental Phenomenology: An Introduction. Cambridge, Cambridge University Press.

MORRIS, K. J. (2015): Merleau-Ponty and Medical Anthropology. Journal of the Anthropological Society of Oxford Online. VII(3), pp 339-354.

OVERGARD, S. y ZAHAVI, D. (2009): "Phenomenological sociology: the subjectivity of everyday life", en M.H. Jacobsen (ed.), Encountering the Everyday: An Introduction to the Sociologies of the Unnoticed. London, Palgrave Macmillan, pp. 93-115.

POTESTÀ, A. (2013): El origen del sentido: Husserl, Heidegger, Derrida. Santiago de Chile, Ediciones Metales Pesados.

PRIEST, S. (1998), Merleau-Ponty. London, Routledge.

REYNOSO, C. (1998): Corrientes en antropología contemporánea. Buenos Aires, Biblos.

REYNOSO, C. (2015): Lenguaje y pensamiento: Tácticas y estrategias del relativismo linguístico. Buenos Aires, Sb editorial.

RICOEUR, P. (2000): "Fenomenología y hermenéutica", Revista electrónica Análisis, 25, pp. 189-207.

RIZO, M. (2015): “Construcción de la realidad, Comunicación y vida cotidiana - Una aproximación a la obra de Thomas Luckmann", Intercom, Rev. Bras. Ciênc. Comun., 38 (2), pp. 19-38. Recuperado de: http://dx.doi. org/10.1590/1809-5844201522.

SAN MARTíN, J. (1994): La fenomenología como una teoría de racionalidad fuerte: estructura y función de la fenomenología de Husserl y otros ensayos. Madrid, Universidad Nacional de Educación a Distancia, UNED.

SAN MARTÍN, J. (2015): "Intersubjetividad, Interculturalidad y Política”, Thémata Revista de Filosofía, 52, pp. 129-145.

SCHÜTZ, A. (1968): El Problema de la Intersubjetividad Trascendental en Husserl. Buenos Aires, Paidós.

SCHÜTZ, A. (1974): El Problema de la Realidad Social. Buenos Aires, Amorrortu.

SCHÜTZ, A. (1993): La construcción significativa del mundo social. Introducción a la sociología comprensiva. Barcelona, Paidós.

THROOP, C. J., y Murphy, K. (2002): “Bourdieu and phenomenology: A critical assessment", Anthropological theory, 2 (2), pp. 185-207.

THROOP, C. J. (2010): Suffering and sentiment: Exploring the vicissitudes of experience and pain in Yap. California, University of California Press. 
TOLEDO, N. (2009): “El Programa Socio-Fenomenológico de Investigación”, Revista Cinta Moebio, 35, pp. 67-87.

ULIN, R. (1990): Antropología y Teoría Social. México, Siglo XXI Editores.

VELASCO, A. (2012): "Hermenéutica y Ciencias Sociales” en E. de la Garza y G. Leyva (eds.), Tratado de metodología de las ciencias sociales: perspectivas actuales. México, Fondo de Cultura Económica, pp. 210-235.

VELOZO, R. (2006): "El “Espíritu comunitario" en el último Husserl", Revista Filosofía, educación y Cultura, 11, pp. 11-29.

VERMEULEN, H. (2015): Before Boas: The Genesis of the Ethnography and Ethnology in the German Enlightenment. Lincoln/London, University of Nebraska Press.

WALDENFELS, B. (1997): De Husserl a Derrida, Introducción a la fenomenología. España, Paidós.

WRIGHT, P. (2003): “Experiencia, intersubjetividad y existencia. Hacia una teoría-práctica de la Etnografía", RUNA, archivo para las ciencias del hombre, 21(1), pp. 347-380. Recuperado en: <http://revistascientificas.filo.uba.ar/ index.php/runa/article/view/1406>.

WRIGHT, P. y CERIANI, C. (2007): "Antropología simbólica: pasado y presente", Relaciones de la Sociedad Argentina de Antropología, 32, pp. 319-348.

ZIRIÓN, A. (2015): "Prólogo" en "Investigaciones Fenomenológicas". Sociedad Española de Fenomenología y Departamento de Filosofía y Filosofía Moraly Política (UNED), 12, pp. 209-220. 